\title{
REVISIÓN
} sanus

\section{Autoeficacia de cuidadores familiares de niños/as con Trastornos del Espectro Autista: revisión integrativa}

\author{
Ramírez-Ochoa, Jonathan ${ }^{\text {**; }}$ Orellana-Yáñez, Alda²
}

\begin{abstract}
RESUMEN
Introducción: Los trastornos del espectro autista en niños/as, generan un efecto negativo en sus cuidadores, donde la comprensión de las cogniciones parentales, como la percepción de autoeficacia, podría afectar el cuidado que entregan. Objetivo: Identificar como la percepción de autoeficacia del cuidador familiar de un niño/a con trastorno del espectro autista, modifica el cuidado que entrega Métodos: Revisión integrativa, realizada en cuatro bases de datos, utilizando las palabras claves, cuidadores, autoeficacia y niño, con booleano AND; filtros, por año e idioma. Resultados: Se seleccionaron 41 artículos, los cuales posterior al análisis y verificación del cumplimiento de criterios de inclusión y exclusión, permitió trabajar con 7 artículos que incorporaban a cuidadores familiares de niños/as con trastorno autista y su percepción de autoeficacia. Conclusiones: El cuidador familiar, debe ser considerado un componente esencial del sistema de cuidado, donde el fortalecimiento de la percepción de autoeficacia, permite que puedan sentirse capaces y empoderados en su rol, favoreciendo su afrontamiento y adquisición de herramientas para desarrollar el cuidado.
\end{abstract}

Palabras clave: Autoeficacia; cuidadores; niño; trastorno del espectro autista; trastorno autístico; enfermería (DeCS; BIREME).

${ }^{1}$ Magister(c) en enfermería, Colaborador docente, Departamento materno-Infantil, Facultad de Enfermería, Universidad de Concepción, Chile.E-mail:jonaramirez@udec.cl

2Doctora en Enfermería. Enfermera Pediatra. Profesor asociado. Directora del Departamento materno-infantil. Director especialidad en enfermería infantil. Facultad de Enfermería. Universidad de Concepción, Chile. E-mail: aorellan@udec.cl

*Autor para correspondencia

Cómo citar este artículo

Ramírez-Ochoa J, Orellana-Yáñez A. Autoeficacia de cuidadores familiares de niños/as con Trastornos del Espectro Autista: revisión integrativa. Sanus. 2018;3(7): 24-39. [Acceso_____ ]; Disponible en: mes día año 


\section{INTRODUCCIÓN}

Los Trastornos del Espectro Autista (TEA), conocido también por sus siglas en inglés como ASD, afectan en promedio a 1 de cada 160 niños en el mundo, con cifras que llegan hasta 1 cada 68 en Estados Unidos, sin tener cifras actualizadas en Chile, definido como un grupo de trastornos caracterizados por alteraciones cualitativas características de la interacción social, las formas de comunicación y por un repertorio repetitivo, estereotipado y restrictivo de intereses y actividades, representado por características generalizadas del comportamiento del individuo en todas las dimensiones de su vida social, familiar y relacional. En la mayoría de los casos el desarrollo es anormal desde la etapa preescolar y sólo en contadas excepciones, las anomalías se manifiestan por primera vez después de los cinco años de edad, sin embargo, es habitual que exista algún grado de alteración cognoscitiva general, aunque estos trastornos están definidos por la desviación del comportamiento en relación a la edad mental del niño (1-3).

El año 2011 se publica en Chile la Guía de Práctica Clínica de Detección y Diagnóstico Oportuno de los Trastornos del Espectro Autista (TEA), la cual plantea un modelo de acción para el rango etario entre los 0 y los 4 años 11 meses, donde durante la evaluación del desarrollo psicomotor en atención primaria, es posible identificar signos de retraso en las áreas de lenguaje y social, lo cual orientará a la aplicación del Cuestionario de Autismo en la Infancia- Modificado (M-CHAT) junto a intervenciones psicosocial de acompañamiento, para posteriormente ser derivado a control con neurólogo infantil quien realiza la confirmación diagnostica, estratificación dentro del nivel de funcionamiento, junto a la identificación de las comorbilidades existentes dentro del nivel secundario y terciario de atención (3).

En caso de que las comorbilidades asociadas al TEA supongan la necesidad de atención con al menos 4 especialista por más de 12 meses, en conjunto a la necesidad de alimentación especial, asistencia tecnológica, rehabilitación y/o educación especial, sin ser estas necesariamente consecuencias del trastorno, es posible ingresarlos dentro del modelo de atención y clasificación de niños y adolescentes con necesidades especiales de atención en salud (NANEAS), que supone un seguimiento mayor en cuanto al tipo y periodicidad de la atención con los distintos profesionales que participan en el programa, tales como médicos especialistas, enfermeras, kinesiólogos, nutricionista, entre otros, en el nivel secundario y terciario de salud (4-6).

El impacto del diagnóstico de un niño/a con TEA en los cuidadores y el subsistema familiar tiene un efecto negativo en los mismos, creando un ciclo de retroalimentación negativa, con una carga emocional y económica para sus familias, con un tipo de cuidado que tiende a ser exigente, demandante y muy desgastante en el tiempo, que debe ser dispensado por personas internas al grupo familiar, generalmente su madre o padre ${ }^{(1,8)}$.

La búsqueda del mejoramiento de la capacidad de los cuidadores para desarrollar el cuidado, se verá reforzada al considerar la salud mental de los padres, así como la comprensión de cómo las cogniciones parentales, tales como la percepción de autoeficacia que poseen, pueden afectar y guiar el proceso de intervención, la habilidad de cuidado que son capaces de ejercer y el cuidado hacia sus hijos ${ }^{(8,9)}$.

El concepto de autoeficacia es introducido inicialmente por Albert Bandura el año 1977, como un pilar en la conformación de la teoría social cognitiva, identifica la imposibilidad de desconocer las interacciones recíprocas entre el ambiente, la persona y la conducta, siendo las dos primeras un molde para la formación de la conducta humana, que en sí misma, se define como, "la manera o forma en cómo los individuos se comportan en su vida y acciones, reconociendo la libertad de este para poder tomar decisiones en salud" (10). La percepción de autoeficacia, es capaz de modificar como los cuidadores familiares piensan, deciden y actúan ${ }^{(11)}$, esto les permite desarrollar las acciones necesarias para obtener resultados esperados en relación al cuidado del niño/a con TEA.

De acuerdo a lo establecido por la Organización Mundial de la Salud (OMS), los cuidadores familiares de niños/as con TEA, son capaces de desarrollar y ejercer un papel fundamental en la prestación de apoyo, lo que ayuda a garantizar su acceso a los servicios sanitarios y educativos, facilitando además, un entorno de soporte y estímulo continuo en cada etapa de su crecimiento y desarrollo, donde en muchas ocasiones, son capaces de brindar de manera directa o ser ejes fundamentales en el proceso de administración de tratamientos psicosociales y conductuales a sus propios hijos (1).

La labor inconmensurable realizada por el cuidador familiar, al ser respaldada y monitorizada por el profesional de enfermería, permite la formulación de herramientas y conocimiento que los direccione en el cuidado. Para esto, enfermería utiliza el respaldo teórico implícito en cada una de las teorías y modelos de enfermería, tomando importancia para esta revisión el Modelo de Promoción de la Salud (MPS) de Nola Pender, desprendido del trabajo de Bandura, quien expone de manera amplia los factores relevantes que intervienen en la modificación de la conducta de una persona, entre ellos la percepción de autoeficacia, dando lugar a las actitudes y motivaciones que estos tienen hacia la acción de promoción de salud y la generación de nuevas conductas sobre sí mismos y sobre las personas que cuidan ${ }^{(12)}$. Este proceso de desarrollo, por el hecho de ser constante y evolutivo, requiere mantener conocimientos actualizados y consensuados que, mediante la revisión 
Cuadro 1. Palabras claves y descriptores indexados

\begin{tabular}{|l|l|l|}
\hline Palabras claves & MeSH & DeCS \\
\hline Cuidadores familiares & Caregivers & $\begin{array}{l}\text { Cuidadores } \\
\text { Cuidadores }\end{array}$ \\
\hline Cuidado & - & - \\
\hline Percepción de autoeficacia & SelfEfficacy & $\begin{array}{l}\text { Autoeficacia } \\
\text { Autoeficácia }\end{array}$ \\
\hline Niño/a & Child & $\begin{array}{l}\text { Niño } \\
\text { Criança }\end{array}$ \\
\hline $\begin{array}{l}\text { Trastorno del espectro } \\
\text { autista }\end{array}$ & $\begin{array}{l}\text { Autistic Disorder } \\
\text { Autism Spectrum Disorder }\end{array}$ & $\begin{array}{l}\text { Trastorno Autístico } \\
\text { Transtorno Autístico }\end{array}$ \\
\hline
\end{tabular}

Fuente: Elaboración propia

integrativa inmersa en la práctica de enfermería basada en la evidencia, entrega a los profesionales evidencia que fundamente, guíe, brinde autonomía y evite la variación en su quehacer ${ }^{(13)}$.

En base a lo mencionado anteriormente, esta revisión integrativa tuvo como propósito identificar como la percepción de autoeficacia del cuidador familiar, es capaz de modificar el cuidado que desarrollan los cuidadores familiares de niños/as con TEA.

\section{MÉTODOLOGÍA}

Entre los meses de septiembre 2017 y enero del año 2018, se realizó una investigación secundaria con metodología de revisión integrativa en las bases de datos Scientific Electronic Library Online (SciELO), Biblioteca Virtual en Salud (BVS), Web Of Science (WOS) y US National Institutes of Health (PubMed), que respondiera a la pregunta ¿Cómo la percepción de autoeficacia del cuidador familiar de un niño/a con trastorno del espectro autista, modifica el cuidado que entrega? Luego se identificaron las palabras claves dentro de la pregunta a utilizar: Cuidadores familiares, cuidado, percepción de autoeficacia y niño/a con trastorno del espectro autista, las que correspondieron según descriptores indexados en DeCS (Descriptores en Ciencias de la Salud) y MeSH (Medical Subject Headings), a los siguientes términos en español, inglés y portugués. (Cuadro 1).

Definidos los descriptores a utilizar, se procedió a identificar los criterios de inclusión y exclusión (Cuadro 2), los cuales tuvieron relación con el artículo mismo y con los resultados de la investigación mostrados en él, en cuanto al tema y población de la revisión integrativa. Se incluyó dentro de esta evaluación, el someter a cada artículo seleccionado a los criterios de identificación de autores, titulo, año, lugar de estudio, revista donde se encuentra

Cuadro 2. Criterios de inclusión y exclusión

\section{Criterios de inclusión}

Edad del niño/a (2 - 12 años)

Publicado desde 2014 a 2018

En idioma inglés, español y portugués

Artículos originales con metodología cuantitativa o cualitativa

Artículos con alto cumplimiento de criterios de calidad metodológica

Revisiones sistemáticas e integrativas

Criterios de exclusión

Artículos Incompletos

Artículos con costo

Artículos con bajo cumplimiento de criterios de calidad metodológica

Fuente: Elaboración propia 
publicado, base de datos indexado, pagina web, palabras claves, objetivo, metodología, resultados y conclusiones, donde la ausencia de claridad o información en alguno de los puntos anteriormente fue causa de eliminación inmediata por no cumplir con los criterios de evaluación metodológica.

Una vez definidos los aspectos preliminares, se realiza ingreso a SciELO, donde el orden de búsqueda utilizado fue Autoeficacia AND Cuidadores AND Niños en español, inglés y portugués respectivamente, sin obtención de resultados positivos. Posteriormente se realizó búsqueda con los primeros dos descriptores y el booleano AND, proporcionando un total de 13, 15 y 15 artículos en cada idioma. Se aplicaron posteriormente criterios de inclusión de título, año y lectura de resumen, y se seleccionan 4 artículos en español y 3 en inglés.

Posteriormente, se ingresó a BVS, WOS y PubMed; se realizó búsqueda avanzada con los tres descriptores en el orden anteriormente usado en SciELO y se aplicó filtro de idioma y año con un resultado de 84, 278 y 140 artículos respectivamente. Se realizó lectura de título y posteriormente resumen, con un total de 11, 12 y 11 artículos seleccionados respectivamente.

Los 41 artículos, son íntegramente leídos en la búsqueda de obtener información respecto del objetivo de la investigación y se realizó un análisis crítico y confirmación de los criterios de inclusión y exclusión, además de la aplicación de los criterios de evaluación metodológica de los articulo y se seleccionan 7 artículos para la realización de esta revisión (Figura 1).

\section{RESULTADOS}

Respecto a la distribución por años de los artículos seleccionados, tres son del $2014^{(10-12)}$, dos del año 2015 ${ }^{(13,14)}$ y dos del año $2017^{(11,16)}$ sin encontrar a la fecha de la investigación en las bases de datos, artículos disponibles para el año 2018. En la distribución por continente de los artículos seleccionados, tres son de América y cuatro de Asia, de los cuales uno fue desarrollado en Brasil, dos en China, uno en India y uno en Pakistán. En Europa se encontraron dos artículos, uno en España y otro en los Países bajos. Con relación al diseño de investigación de los artículos, se obtuvo: cinco de tipo

Figura 1. Metodología de búsqueda y selección de artículos incluidos en revisión integrativa

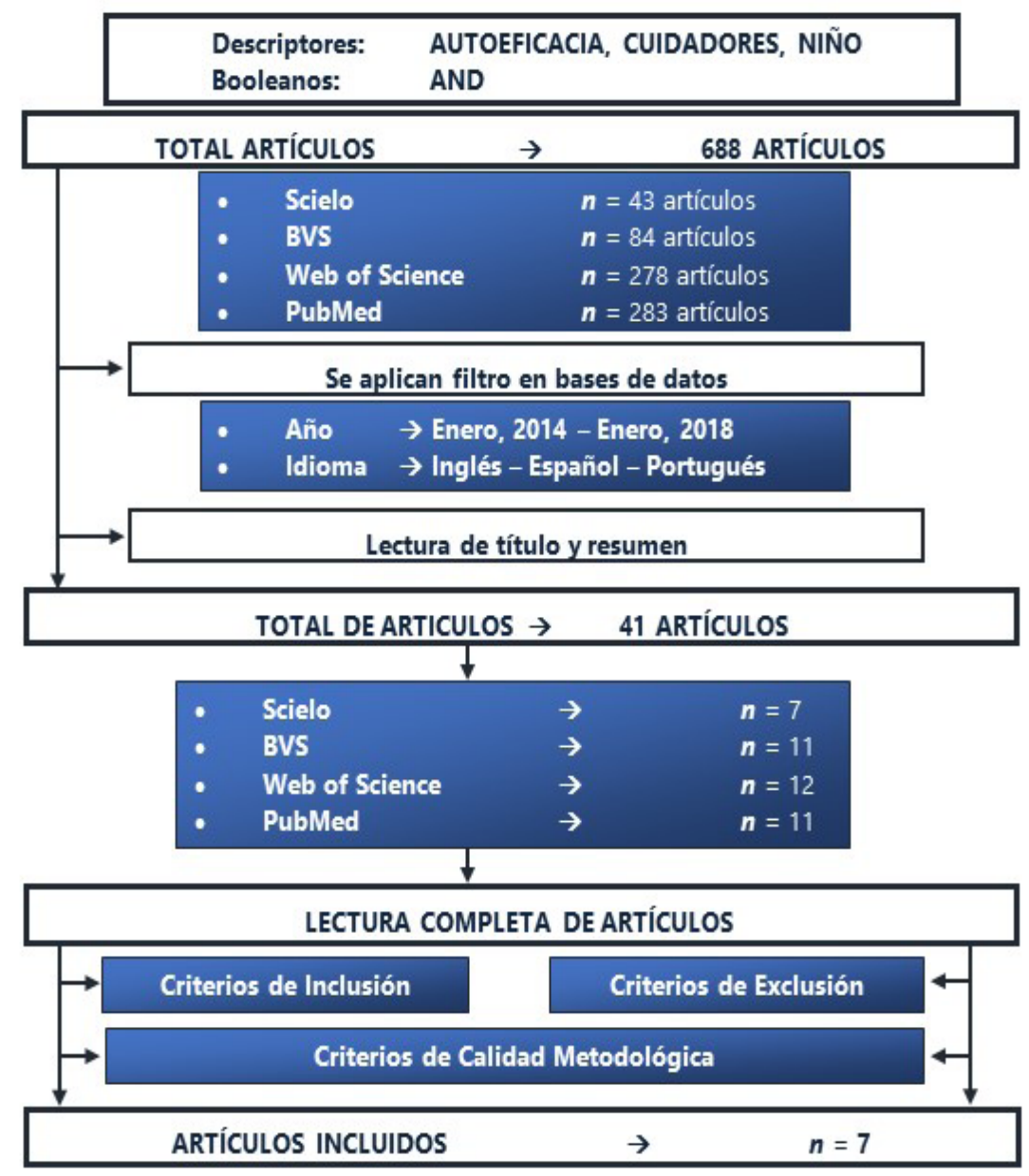

Fuente: Elaboración propia 
descriptivo correlacional, uno cuasi experimentales y uno experimental.

De los diez artículos seleccionados, se identifican tres en PubMed ${ }^{(14,18,19)}$, tres en WOS ${ }^{(15,16,17)}$ y uno en BVS (11).

El cuadro 3, detalla cada artículo seleccionado para esta revisión, en orden cronológico de publicación, lo que permitió establecer una lectura comparativa entre ellos, contrastando objetivos, resultados y conclusiones obtenidos por cada autor.

\section{DISCUSIÓN}

Dentro de los artículos considerados para esta revisión, uno se encuentra indexado en una revista de Enfermería (14), seis en distintas revistas del área de psicología (14, 1619). Los artículos seleccionados, muestran que, dentro de las características del cuidador familiar, es la mujer quien predominantemente desarrolla el cuidado de los niños/ as con TEA ${ }^{(14-16,19)}$, quien debe cumplir una función para la cual no han sido formadas ni guiadas y sin dejar de desempeñar otros roles asignados socialmente, como ser esposa, madre de otros niños, organizar el hogar, entre otros. Se identifica, además, una preferencia hacia el abandono de su fuente laboral, para dedicarse a tiempo completo al cuidado $(14,16)$. Lo anterior, nos guía a la idea de abnegación que sufren como cuidadoras familiares, postergándose como personas, en cada uno de sus proyectos individuales y familiares, sacrificando muchas veces su propia salud mental, lo que favorece en el tiempo el desarrollo de estrés parental asociado al cuidado, aumento del nivel de percepción de sobrecarga como cuidador y una disminución en la comprensión del cuidado que deben ejercer y como este se hace coherente con su propia vida ${ }^{(16)}$.

La literatura analizada, reconoce como factores de riesgo para un deterioro de la salud mental de los cuidadores familiares, la baja calidad de relación de pareja que poseen, el bajo nivel de resiliencia, ser cuidador de más de un niño con TEA, la ausencia de apoyo social percibido, la baja percepción de autoeficacia, la severidad y gravedad de los síntomas del tipo de alteración específico del niño con TEA y se destaca entre estos factores, la percepción de autoeficacia y la gravedad de deterioro del niño como predictores significativos de estrés parental ${ }^{(17)}$.

Una baja percepción de autoeficacia se relaciona directamente con la imposibilidad que poseen los cuidadores familiares para descansar, con un aumento del nivel ansiedad y de sobrecarga del cuidador, disminución de la calidad de vida relacionada con salud mental, del locus control y del nivel de resiliencia (16). Adicionalmente la percepción de autoeficacia, es la determinante intrínseca que mejor permite comprender cómo las madres de niños/as con TEA se sienten capaces frente al cuidado que desempeñan, permitiéndoles poseer una mayor expresión de sentimientos en conjunto a la generación y reconocimiento de un entorno que favorezca el apoyo social; para los padres, cómo cuidador familiar el poder controlar y solucionar situaciones que signifiquen problemas para ellos o el núcleo familiar, estaría favorecido por un buen nivel de autoeficacia ${ }^{(18)}$.

Al generar intervenciones que incluyan tanto al equipo profesional como a los cuidadores familiares, donde se les enseña a afrontar la crisis, ayuda a que los cuidadores familiares, puedan empoderarse y fortalecer su propia percepción de autoeficacia, sintiéndose útiles dentro del sistema de atención, lo que favorece la calidad de vida relacionada con la salud mental que poseen, generando una mayor percepción de confianza en las decisiones y acciones que toman, así como de competencia y capacidad para cuidar ${ }^{(14,15)}$.

Es necesario que los profesionales encargados de trabajar y abordar en cualquier etapa de desarrollo tanto al niño/a con TEA, sean capaces de establecer asociaciones colaborativas con las familias, centrándose en la capacitación de los cuidadores familiares, incluyendo a estos últimos, como parte integral del equipo que trabaja en pos del desarrollo e integración del niño/a con TEA, proceso que se verá favorecido al facilitar el desarrollo de su propia percepción de autoeficacia como cuidador, lo cual fortalecerá el bienestar personal y familiar, mediante la sinergia establecida con el cuidado que desarrollan ${ }^{(15,19)}$.

\section{CONCLUSIONES}

En la actualidad, el modelo de atención, del cual está impregnada la totalidad de la guía clínica disponible en Chile, se centra en el manejo del niño/a con TEA, restando importancia al valor agregado que supone el cuidador sobre ellos, aun con falencias en la entrega de directrices especificas respecto al manejo y las acciones que deben tomar los cuidadores en las distintas etapas que deben vivir junto a sus hijos, con el fin de facilitar su afrontamiento y adaptación a esta nueva vida, a cargo de un niño con esta condición, a no ser que categoricen por alguna condición crónica concomitante o un una condición severa dentro de los TEA que los permita integrar parte del programa NANEAS (3-6).

La falta de inclusión y acompañamiento de los cuidadores familiares en esta tarea, llena de desafíos, miedos y deberes, tanto desde el punto de vista individual como familiar, se traduce en un descuido hacia ellos y una falta de consideración a las necesidades individuales que pudiesen surgir tras el diagnóstico de sus hijos.

El cuidador familiar, frente al fortalecimiento de la autoeficacia que percibe sobre sí mismo, logra mejorar su propia calidad de vida, lo cual repercute positivamente tanto en su proyecto de vida individual como familiar, 


\begin{tabular}{|c|c|c|c|c|c|c|c|}
\hline & 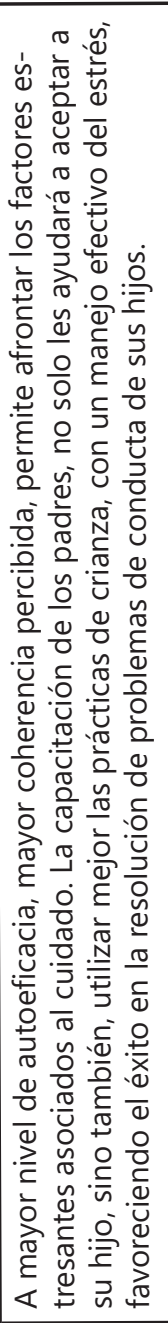 & 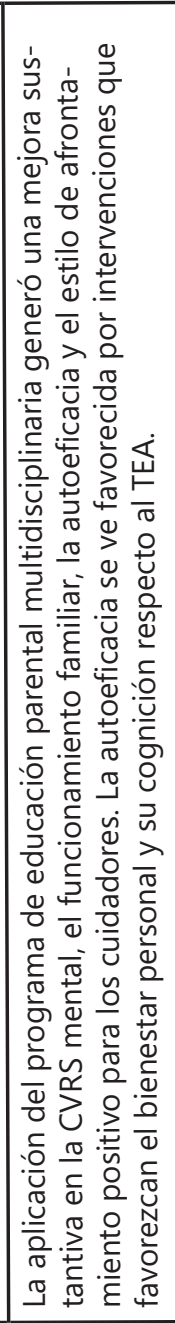 & 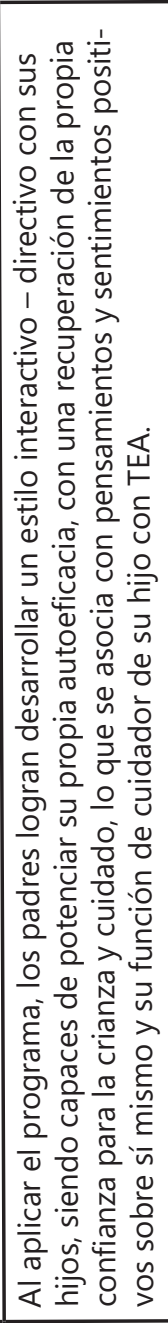 & 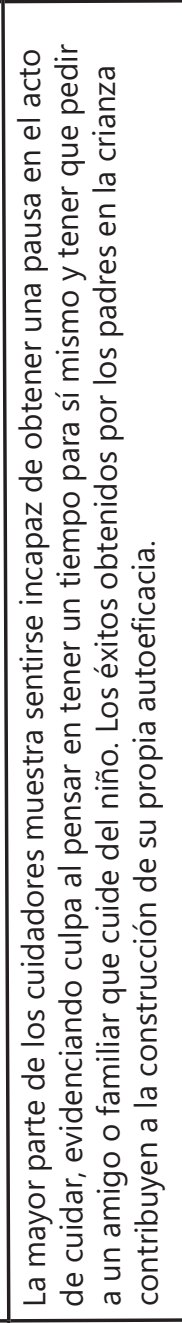 & 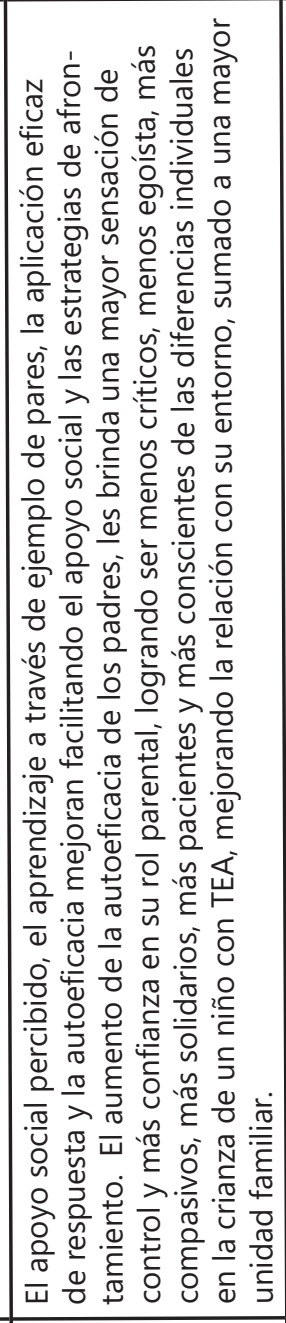 & 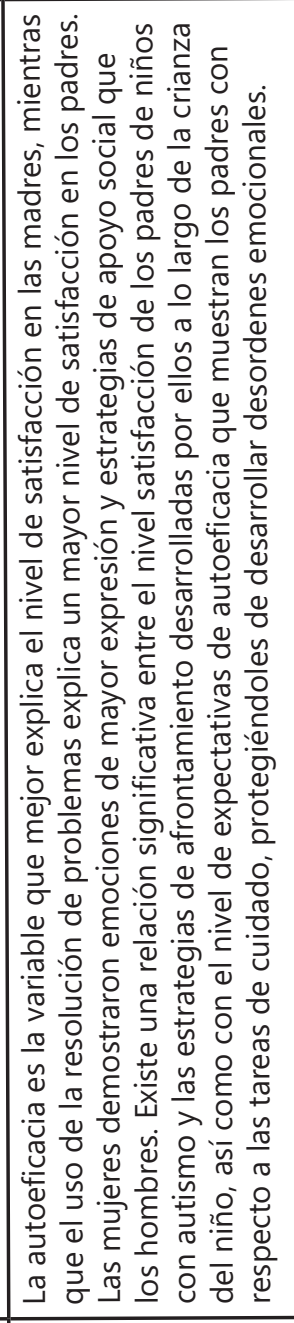 & 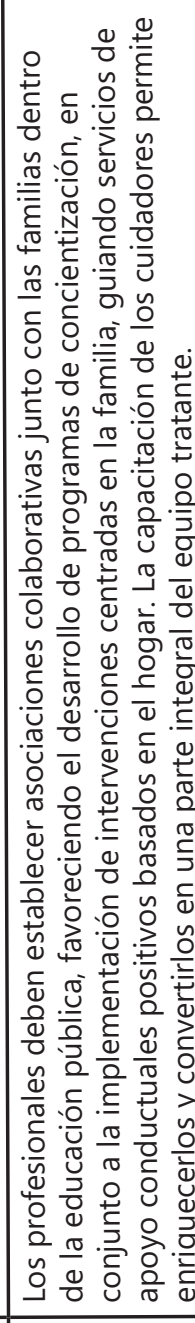 \\
\hline 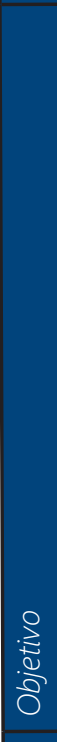 & 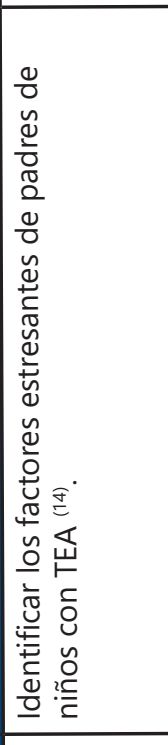 & 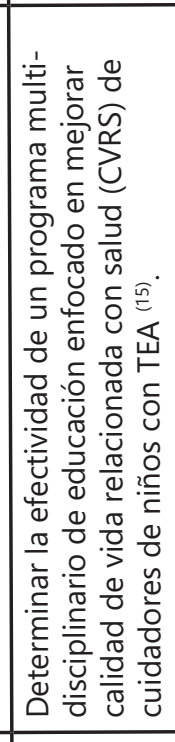 & 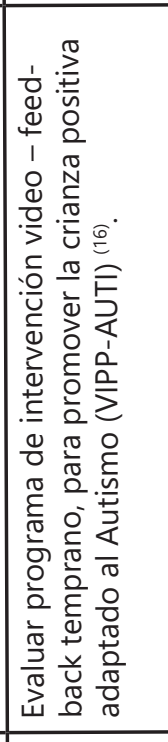 & 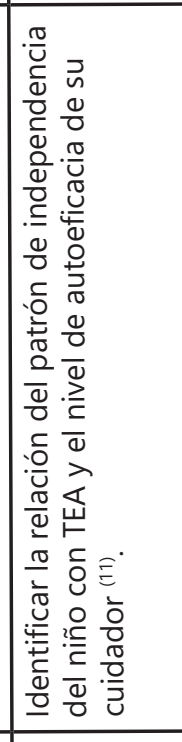 & 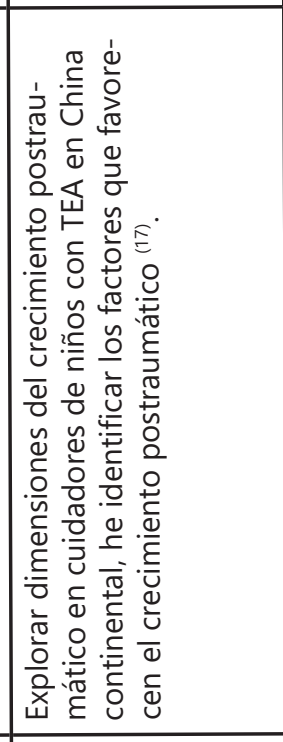 & 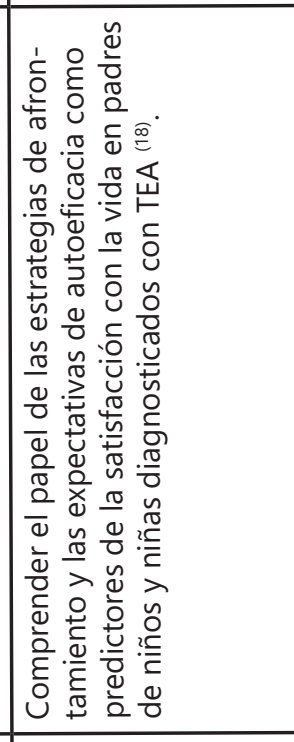 & 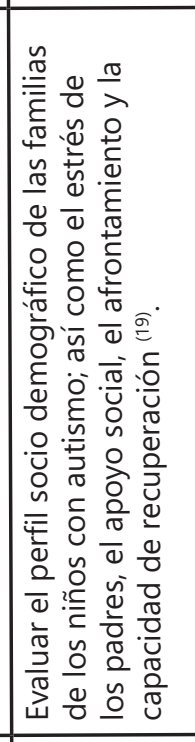 \\
\hline 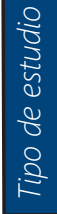 & 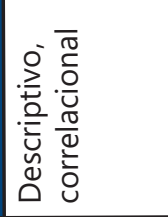 & 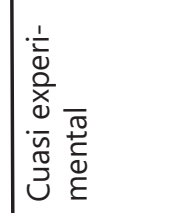 & 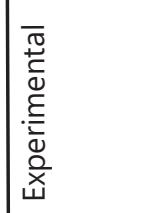 & 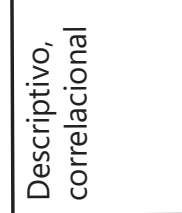 & 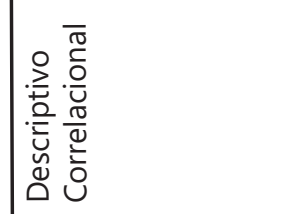 & 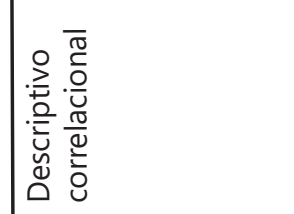 & 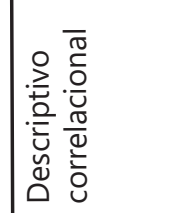 \\
\hline
\end{tabular}


además de entregar herramientas para ser protagonista en el proceso y accionar necesario para cuidar al niño con TEA. Facilitar la generación de instancias que potencien la autoeficacia de los padres o familias es crucial, al aumentar el bienestar de los cuidadores y entregar el valor agregado de hacerlos capaces y empoderados de su nuevo rol de cuidador, donde se perciban capaces de poseer habilidad para desarrollar el cuidado, generando un impacto tanto en la vida del niño/a con TEA, como en las decisiones que tomarán a lo largo de sus vidas.

Considerar al cuidador como un componente esencial del subsistema de cuidado, exige aceptar su potencial y sus limitaciones, y obliga a entregarles las herramientas necesarias para mejorar su habilidad de cuidado en términos de incrementar la capacidad de autoeficacia y afrontamiento, de manera tal, que sea capaz de satisfacer las necesidades de la persona cuidada, responda a la problemática familiar, mantenga su propia salud y fomente la buena relación cuidador persona cuidada, siendo capaz de reconocer y establecer apoyos formales e informales, manejo de sentimientos de inadecuación o culpa y planeación de su propio futuro y el de su grupo familiar ${ }^{(20)}$.

Enfermería, después de responder al objetivo de esta revisión integrativa debería formular estrategias, programas de intervención, centradas en el cuidador familiar de niños/as con TEA, contextualizado dentro de la Atencion Primaria en Salud en cooperación continua con otras disciplinas mediante el MPS (12), buscando identificar las características y experiencias individuales del cuidador familiar que repercuten en la conformación de su percepción de autoeficacia para desarrollar el propio cuidado y el del niño, en la búsqueda de lograr el desarrollo integral del grupo familiar, reforzado por la consecución de logros asociados al cuidar.

\section{REFERENCIAS BIBLIOGRAFICAS}

1.Organización mundial de la Salud. Trastorno del espectro autista [Internet]. Ginebra, Suiza, 2017. [Citado 31 de Ago, 2018] Disponible en: http://www.who.int/ es/news-room/fact-sheets/detail/autism-spectrumdisorders

2.Centros para el control y la prevención de enfermedades. Trastornos del espectro autista (TEA) [Internet]. Druid Hills, Estados Unidos, 2016. [Citado 31 de Ago, 2018] Disponible en: https://www.cdc.gov/ncbddd/spanish/ autism/index.html

3.Ministerio de Salud. Guía de práctica clínica: Detección y diagnóstico oportuno de los trastornos del espectro autista. [Internet]. Santiago, Chile 2011.

[Citado18deDic,2016]Disponibleen:http://web.minsal.cl/ portal/url/item/bd81e3a09ab6c3cee040010164012ac2. pdf
4.Ministerio de Salud, Gobierno de Chile. Programa nacional de salud de la infancia con enfoque integral [Internet]. Santiago, Chile 2013. [Citado 31 de Ago, 2018] Disponible en: https://diprece.minsal.cl/wrdprss minsal/wp-content/uploads/2015/10/2013_ProgramaNacional-de-Salud-de-la-infancia-con-enfoque-integral. pdf

5.Ministerio de Salud, Gobierno de Chile. Norma Técnica para la supervisión de niños y niñas de 0 a 9 años en la Atención Primaria de Salud: Programa Nacional de Salud de la Infancia. Santiago, Chile 2014. [Citado 31 de Ago, 2018] Disponible en: http://www.minsal.cl/sites/default/ files/files/2014_Norma\%20T\%C3\%A9cnica\%20para\%20 la\%20supervisi\%C3\%B3n\%20de\%20ni\%C3\%B1os\%20 y\%20ni\%C3\%B1 as\%20de\%200\%20a\%209\%20en\%20 APS_web(1).pdf

6.Flores J, Lizama M, Rodriguez N, Ávalos M, Galanti M, Barja S, et al. Modelo de atención y clasificación de niños y adolescentes con necesidades especiales de atención en salud-NANEAS: Recomendaciones del comité NANEAS de la Sociedad Chilena de Pediatría. Rev Chil Pediatr. 2016; 87 (3): 224-32.

7.Organización Mundial de la Salud. Trastorno del espectro autista. Nota descriptiva. [Internet] enero de 2016. [Citado 19 de diciembre, 2016] Disponible en: http://www.who.int/mediacentre/factsheets/autismspectrum-disorders/es/

8.Organización Mundial de la Salud. Preguntas y respuestas sobre los trastornos del espectro autista. Reportajes. Archivo de preguntas y respuestas. [Internet] abril 2016. [Citado 18 de diciembre, 2016] Disponible en: http://www.who.int/features/qa/85/es/

9.Karst J, Van Hecke A. Parent and Family Impact of Autism Spectrum Disorders: A Review and Proposed Model for Intervention Evaluation. Clin Child Fam Psychol Rev [Internet] 2012; 15(3):247-77. [Citado 24 de diciembre, 2016] Disponible en: https://link.springer. com/article/10.1007\%2Fs10567-012-0119-6

10.Brockman M, Hussain K, Sanchez B, Vueltas B. Managing Child Behavior Problems in Children with Autism Spectrum Disorders: Utilizing Structural and Solution Focused Therapy with Primary Caregivers. Am J Fam Ther [Internet]. 2016; 44(1): 1-10. [Citado 10 de Septiembre, 2018] Disponible en: http://www.tandfonline. com/doi/full/10.1080/01926187.2015.1099414

11.Bandura A. Comments on the crusade against the causal efficacy of human thought. J Behav Ther Exp Psychiatry. [Internet]. 1995. [Citado 10 de mayo, 2016] 26(3):179-190. Disponible en: https://www.uky. edu/ eushe2/Bandura/Bandura1995JBTEP.pdf 
12.Tabaquim M, Vieira R, Razera A y Ciasca S. Autoeficácia de cuidadores de crianças com o transtorno do espectro autista. Revista Psicopedagogia, [Internet].

2015. [Citado 26 de Ago, 2018]; 32 (99): 28592. de Disponible en: http://pepsic.bvsalud. org/scielo.php?script =sci_arttext \&pid=S0103$84862015000300002 \& \operatorname{lng}=$ pt\&tlng $=$ pt.

13.Sakraida T. Nola J. Pender: Modelo de promoción de la salud. En: Raile M. Modelos y teorías en enfermería. Octava edición. Ediciones Elsevier. 2015; Unidad IV, cap 21: 385-404.

14.Orellana A, Paravic T. Enfermería basada en evidencia: barreras y estrategias para su implementación. Ciencia y Enfermería. [Versión impresa]. 2016; 13(1): 17-24.

15.Shahida S, Sumaira S. Factors Associated with Stress Among Parents of Children with Autism. J Coll Physicians Surg Pak. [Internet]. 2014; 25 (10): 752-6. [Citado 13 de enero, 2017] Disponible en: https://www.ncbi.nlm.nih. gov/pubmed/26454393

16.Ji B, Sun M, Yi R, Tang S. Multidisciplinary Parent Education for Caregivers of Children with Autism Spectrum Disorders. Arch Psychiatr Nurs. [Internet]. 2014; 28: 319-26. [Citado 4 de ene, 2017] Disponible en: https://www.sciencedirect.com/science/article/pii/ S0883941714000958?via\%3Dihub

17.Poslawsky I, Naber F, Bakermans M, Daalen E, Engeland $\mathrm{H}$, ljzendoorn $\mathrm{M}$. Video-feedback Intervention to promote Positive Parenting adapted to Autism (VIPP-AUTI): A randomized controlled trial. Autism. [Internet]. 2014; 19 (5): 588-603. [Citado 4 de enero, 2017] Disponible en: http://aut.sagepub.com/content/ early/2014/06/10/1362361314537124.abstract

18.Merighi M, De Souza $R$, Ribeiro A, Ciasca S. Autoeficácia de cuidadores de crianças com o transtorno do espectro autista. Rev. Psicopedag. [Internet]. 2015; 32(99): 285-92. [Citado 24 de diciembre, 2016] Disponible en: $\quad$ http://pepsic.bvsalud.org/scielo.php?script=sci_ arttext\&pid $=$ S0103-84862015000300002

19.Zhang W, Yan T, Barriball L, While A, Hong X. Post-traumatic growth in mothers of children with autism: A phenomenological study. Autism [Internet]. 2015; 19(1): 29-37. [Citado 7 de enero, 2017] Disponible en: http://aut.sagepub.com/content/ early/2013/11/08/1362361313509732

20.Luque B, Yañez V, Tabernero C, Cuadrado E. The role of coping strategies and self-efficacy as predictors of life satisfaction in a sample of parents of children with autism spectrum disorder. Psicothema. [Internet].2017; 29(1): 55-60. [Citado 2 de enero, 2018] Disponible en: http:// www.psicothema.com/PDF/4363.pdf

21.Das S, Das B, Nath K, Dutta A, Bora P. Impact of stress, coping, social support, and resilience of families having children with autism: A north east India-bases study. Asian J Psychiatr. [Internet]. 2017; 28:133-9 [Citado 07 de enero, 2018] Disponible en: ttps://www.sciencedirect.com/ science/article/pii/S1876201817300217?via\%3Dihub

22.Barrera L, Galvis C, Moreno M, Pinto N, Pinzón M, Romero E, Sánchez B. La habilidad de cuidado de los cuidadores familiares de personas con enfermedad crónica: un estudio comparativo de tres unidades académicas de enfermería. Invest. Educ. Enferm. [Internet].2006; 24(1): 36-46. [Citado 20 de enero, 2017] Disponible en: http://web.minsal.cl/portal/url/item/ bd81e3a09ab6c3cee040010164012ac2.pdf 